\title{
ABSORPTION CORRECTIONS FOR X-RAY TEXTURE MEASUREMENT OF ANY SHAPE SAMPLE
}

\author{
By T. MONTESIN, J.J. HEIZMANN, A. VADON \\ Laboratoire de Metallurgie Physique \& Chimique, \\ METZ University - FRANCE
}

A pole figure needs generally more than 2000 points of measurement, each point corresponding to a specific location of the sample in space. For each position, the detected intensity will depend on :

- the irradiated volume,

- the volumic fraction of cristallites in Bragg position in this volume, this one depending in its turn on the cristallographic texture,

- the absorption of X-Rays by the material (1).

For flat and thick samples with an homogeneous texture, corrections are not required, because there is an exact balance between the increase of intensity due to the diffracting volume and the decrease of intensity due to the absorption (2). The corrections are necessary for an any shape sample.

In a first part, we present the absorption correction for X-ray texture measurement of any shape sample. In a second part, the intensities corrections are applied to a set of wires. the presented experiments demonstrate that the intensity corrections should be used to determine the texture of wires accuratly.

\section{I) TEXTURE MEASUREMENT OF ANY SHAPE SAMPLE}

We used two kinds of texture goniometer : a conventional one, and a multifigures texture goniometer using a curved position sensitive detector (5). With the two kinds of goniometer, we are working in the reflexion mode and using the Shulz goniometric movements to describe the sterographic net (fig. 1). The sample rotates around its azimuthal axis $O x^{\prime}$ ( $\varphi$ rotation) which is normal to the sample and its tilting axis $O y$ ( $\psi$ rotation) which lies in the sample.

At $(\varphi, \psi)$ position, consider a small elementary volume $\mathrm{dV}$ irradiated by the incident $\mathrm{X}$-ray beam (fig. 1 ). The diffracted intensity by this volume is

where

$$
\mathrm{dI}_{\varphi \psi}=\mathbf{i} \cdot \mathrm{K}_{\varphi \psi} \cdot \mathrm{e}^{-\mu \mathrm{t}(\varphi, \psi)} \mathrm{dV}
$$

$-\mathrm{i}=$ the diffracted intensity by an isotropic unit volume located at the surface of the sample.

$-\mu=$ the linear absorption coefficient of the material. 
$-t(\varphi, \psi)=$ the pathway of $X$-ray in the sample at the $(\varphi, \psi)$ position (fig. $1, t=a+b)$

- $K_{\varphi \Psi}=$ the volumic fraction of cristallites in the volume dV fulfilling the Bragg condition at the $(\varphi, \Psi)$ position. $K_{\varphi \psi}$ stands for the orientation factor.

The whole detected intensity or measured intensity at the $(\varphi, \psi)$ position is :

$$
I_{\text {measured }}(\varphi, \psi)=1 . \int_{V_{\varphi \psi}} K_{\varphi \psi} \cdot e^{-\mu t(\varphi, \psi)} d V
$$

Our aim is to find $\mathrm{K}_{\varphi \Psi}$ from $\mathrm{I}_{\text {measured }}(\varphi, \psi)$. Several cases may occur depending on how $\mathrm{K}_{\varphi \Psi}$ can be extracted from the integral.

\section{L1) the sample is isotropic}

$K_{\varphi \Psi}$ does not depend on the sample position $(\varphi, \Psi)\left(K_{\varphi \Psi}=K\right)$

$$
I_{\text {measured }}(\varphi, \psi)=i \cdot K \cdot \int_{V_{\varphi \psi}} e^{-\mu t(\varphi, \psi)} d V
$$

\section{L2) the sample is an homogeneous textured material}

The texture is the same in the whole material. For each given $(\varphi, \psi)$ position, all the elementery volume have the same $K_{\varphi \psi}$ but $K_{\varphi \psi}$ is altered when the position of the sample is changed (fig. 2). So the measured intensity is, for a given $(\varphi, \psi)$ position of the sample :

$$
I_{\text {measured }}(\varphi, \psi)=\mathbf{i} \cdot K_{\varphi \psi} \cdot \int_{V_{\varphi \psi}} e^{-\mu t(\varphi, \psi)} d V
$$

To know $\mathrm{K}_{\varphi \psi}$, the factor $\mathrm{A}_{\varphi \psi}$ needs to be determined :

$$
A_{\varphi \psi}=\int_{V_{\varphi \psi}} e^{-\mu t(\varphi, \psi)} d V
$$

This factor takes into account the diffracting volume $\mathrm{V}_{\varphi \Psi}$ and the pathway of $\mathrm{X}$ rays $t(\varphi, \psi)$. $A_{\varphi \psi}$ can be normalized once we have taken a reference position of the sample. Consider $(\varphi=0, \psi=0)$ the reference position. Then we obtain a dimensionless coefficient

$$
N_{\varphi \psi}=\frac{\int_{V_{\varphi \psi}} e^{-\mu t(\varphi, \psi)} d V}{\int_{V_{00}} e^{-\mu t(0,0)} d V}=\frac{A_{\varphi \psi}}{A_{00}}
$$

so the measured intensity can be written as follows :

$$
I_{\text {measured }}(\varphi, \Psi)=\mathbf{i} \cdot \mathbf{A}_{00} \cdot \mathbf{K}_{\varphi \Psi} \cdot \mathbf{N}_{\varphi \Psi}
$$

In this expression, i. $\mathrm{A}_{00}$ is an experimental constant factor which depends solely on the experimental conditions (size of the beam, 1 , material, shape of the sample,...). The term i. $\mathrm{A}_{00} \mathrm{~N}_{\varphi \psi}$ can be considered as the intensity diffracted by the same but isotropic sample placed under the same experimental conditions; with $N_{\varphi \Psi}$ being the 
factor taking into account the evolution of the diffraction volume and the absorption during the $(\varphi, \psi)$ rotations.

One can define a corrected diffracted intensity which is

$$
I_{\text {corrected }}(\varphi, \psi)=I_{\text {measured }}(\varphi, \psi) / \mathbf{N}_{\varphi \psi}=\mathbf{i} \cdot \mathbf{A}_{00} \cdot \mathbf{K}_{\varphi \psi}
$$

This corrected intensity is directly proportional to the orientation factor $\mathrm{K}_{\varphi \Psi}$.

\section{L3) The sample is an heterogeneous textured material}

The texture is variable in the material; this is the general case in which the orientation factor is not the same in all the irradiated volume at a given position $(\varphi, \psi)$ of the sample (fig. 3).

The orientation factor cannot be extracted from the integral. To be able to extract it, it is necessary to know the orientation factor of each volume dV this being the unknown. In fact, it is impossible to be precise regarding the quantity contributed by each $\mathrm{dV}$ to the diffracted intensity.

Nevertheless the use of the correction factor of the homogeneous sample can give us a mean value of orientation factor $\mathrm{K}_{\varphi \Psi}$ defined by

$$
\overline{K_{\varphi \psi}}=\frac{\int_{V_{\varphi \psi}}^{K_{\varphi \psi}} e^{-\mu t(\varphi, \psi)} d V}{\int_{V_{\varphi \psi}} e^{-\mu t(\varphi, \psi)} d V}
$$

This mean value is calculated from the measured intensity :

$$
\overline{K_{\varphi \psi}}=\frac{I_{\text {measured }}(\varphi, \psi)}{\text { i . } A_{00} N_{\varphi \psi}}
$$

It is close to the true value if the texture evolution across the irradiated volume is not important or if the absorption coefficient is such as to permit the observation of only the surface of the sample.

\section{II) EXPERIMENTAL STUDY OF WIRES}

As the diffracted intensity of a single small diamater wire is to weak, it becomes necessary to measure several wires with the same diameter. As a consequence, it is compulsory to have considerable intensity corrections during the texture measurement, because the pathway of $\mathrm{X}$ rays in the material is complex.

Intensity corrections have already been studied by F. LANGOUCHE and al (3) and at the same time by ourselves (4). The main difference between these two recent studies is that one takes into account the influence of the adjoining wires in the absorption phenomenon (4), while the other fails to do so(3).

\section{II.1) Sample preparation}

The wires are stuck contiguously on a glass plate. The sample is set up on the goniometer. It swings in its own plane perpendicularly to the wires axis. XYZ is a fixed reference chosen to do all the calculations concerning the diffracting volume and the absorption pathway. 
One fig. 4 is represented a cross section of the wires by the incidence plane XOY at a position $(\varphi, \psi)$. In this plane, the $\mathrm{N}_{\varphi \Psi}$ calculation factor is brought down to a ratio of two area integrals.

$$
N_{\varphi \Psi}=\frac{\cos (\varphi) \cdot \int_{S_{\varphi \Psi}} e^{-\mu t(x, y)} d x d y}{\int_{S_{\infty}} e^{-\mu t(x, y)} d x d y}
$$

Where:

- $S_{\varphi \psi}=$ cross area of the wire by the incidence plane.

- dxdy = elementary area.

$-t(x, y)=$ pathway of X-Ray ( incident + diffracted beams) which depends on the coordinates $(x, y)$ of the elementary area. For exemple, in this figure, $t=a+b+c+e+f$.

The $\mathrm{N}_{\varphi \psi}$ calculation is done for the whole cross area of the wire by a microcomputer. It is generally stopped when the pathways $t(x, y)$ of $X$-Rays give an intensity lower by $1 \%$ than that located on the surface. In the case of steel wires observed whith $\lambda \mathrm{FeK} \alpha$, the variation of the correction factor $\mathrm{N}_{\varphi \psi}$ can go from 1 to 10.

On fig. 5, are presented the two correction factors $\mathrm{N}_{\varphi \Psi}$ calculated for a $98 \mu \mathrm{m}$ diamater aluminium wire. The first one take into account the adjoining wires, whereas the second one doesn't. There is great differnces between these two factors.

\section{IL.2) Application of the corrections to wires}

The fig. 6 present respectively the measured (110) pole figure of a $300 \mu \mathrm{m}$ steel wire, the correction factor of pole figure calculated, and the (110) corrected pole figure. One can say that :

1) the correction factor varies from 1 to 4 which is not negligeable for this kind of wire.

2) the absence of the fibrous axis on the measured pole figure which appears perfectly on the corrected pole figure.

\section{III) CONCLUSION}

This studies reveal the absolute necessity of using intensity corrections to get the texture of the wires, which is important to know during the cold drawing process.

The influence of adjoining wires becomes important when the wires are thin and when the absorption coefficient is weak $(\mu \mathrm{R}<3)$

1. International Tables of X ray Diffraction (J.S. Kasper and K. Lonsdasle 1959), Vol. 2

2. A. Guinier, Théorie \& technique de la Radiocristallographie (Paris: Dunod 1956)

3. F. Langouche, E. Aernoudt and P. Van Houte, J. Appl. Cryst., 22,533 (1989)

4. T. Montesin and J.J. Heizmann, Mém. Et. Sci. Rev. Mét., 217, April (1990)

5. J.J. Heizmann and C. Laruelle, J. Appl. Cryst., 19, 467, (1986) 


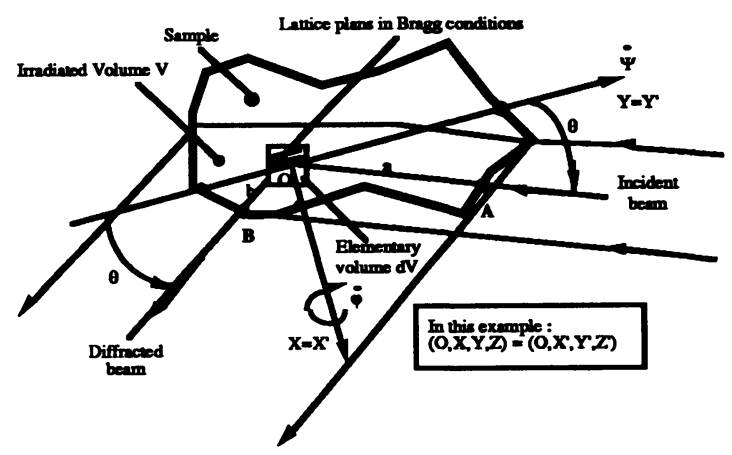

Figure 1:Diffracting volume of an any shape sample irradiated by the incident X-ray beam.

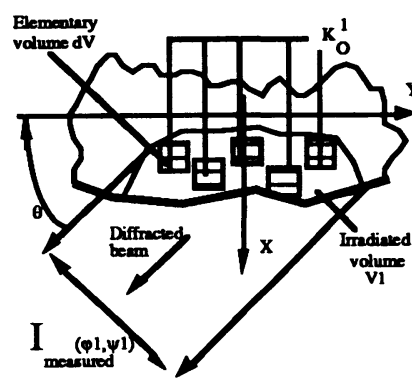

(el.w1) porition of the cumple

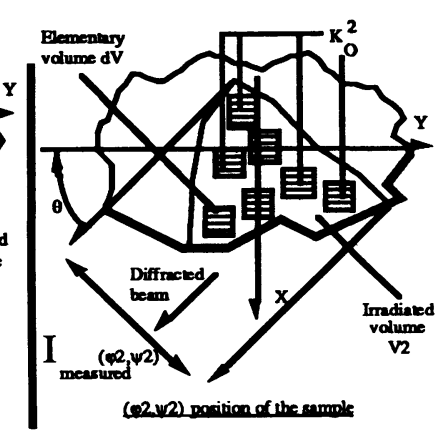

(Q2.12) porition of the sumble

Figure 2 : "Measured intensity" diffracted by a textured and homogeneous material.

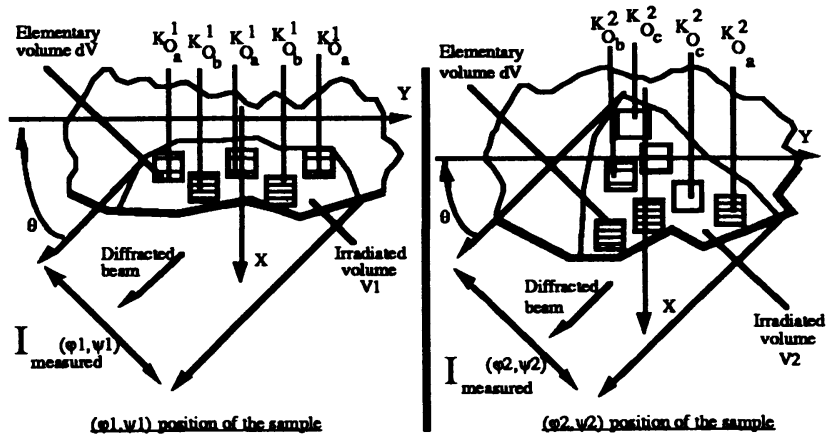

Figure 3 : "Measured intensity" diffracted by a textured and heterogeneous material.

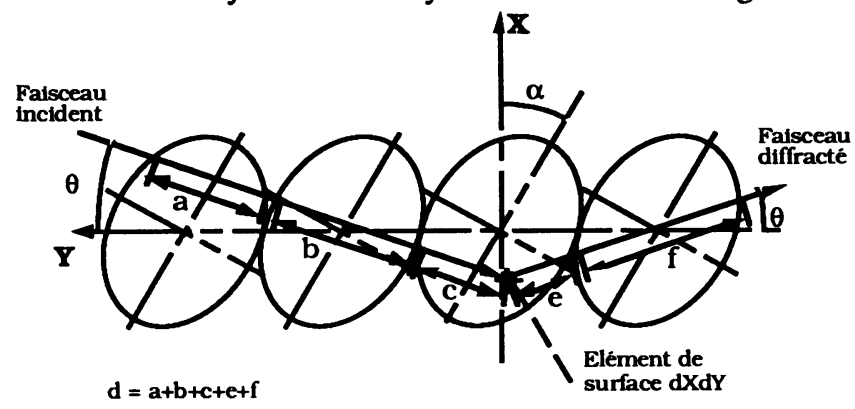

Figure 4: Cross section of the wires by the incidence plane. $t=a+b+c+e+f=$ pathway of the X-ray in the wire 
Fig. 5 :calculated correcting factors for a $98 \mu \mathrm{m}$ aluminium wire, measured with $\lambda=\mathrm{K} \alpha \mathrm{Mo}$. Left $=$ with adjoining wires.
Fig 6 a
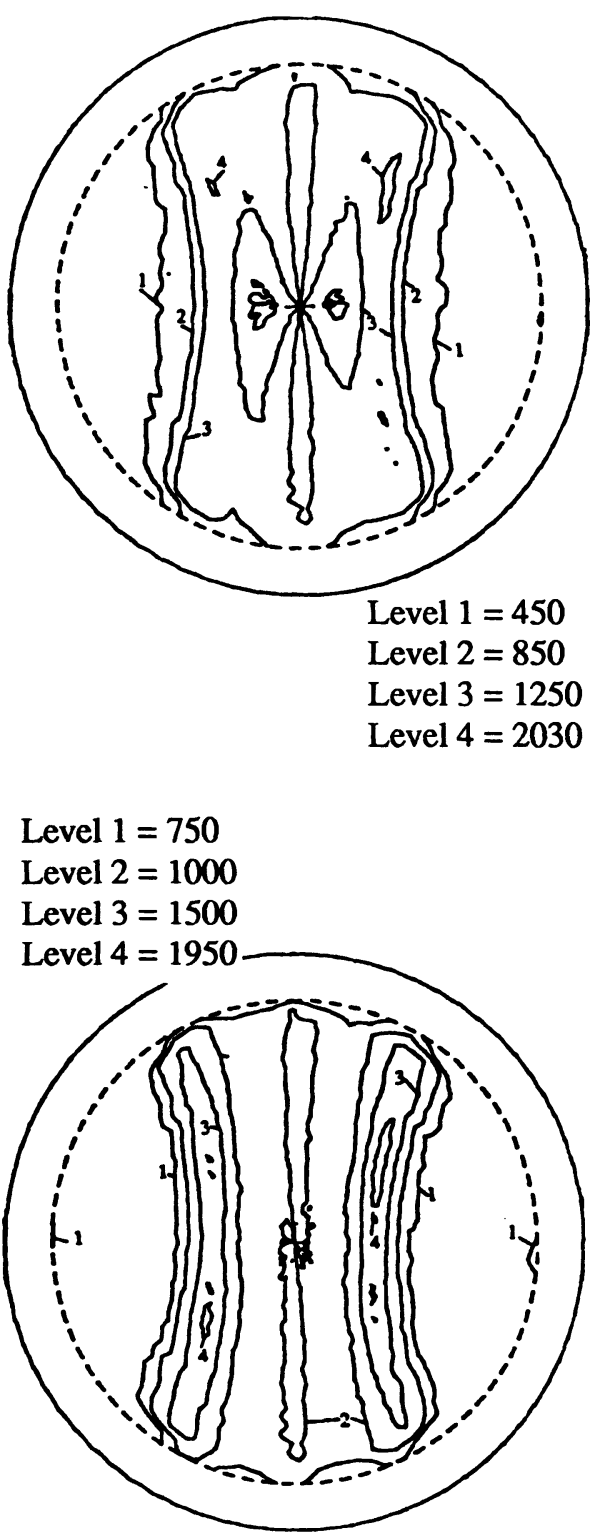

Fig 6 b

Level $1=0,6$

Level $2=0,8$

Level $3=1,0$

Level $4=1,4$

Level $5=1,6$

Level $6=1,9$
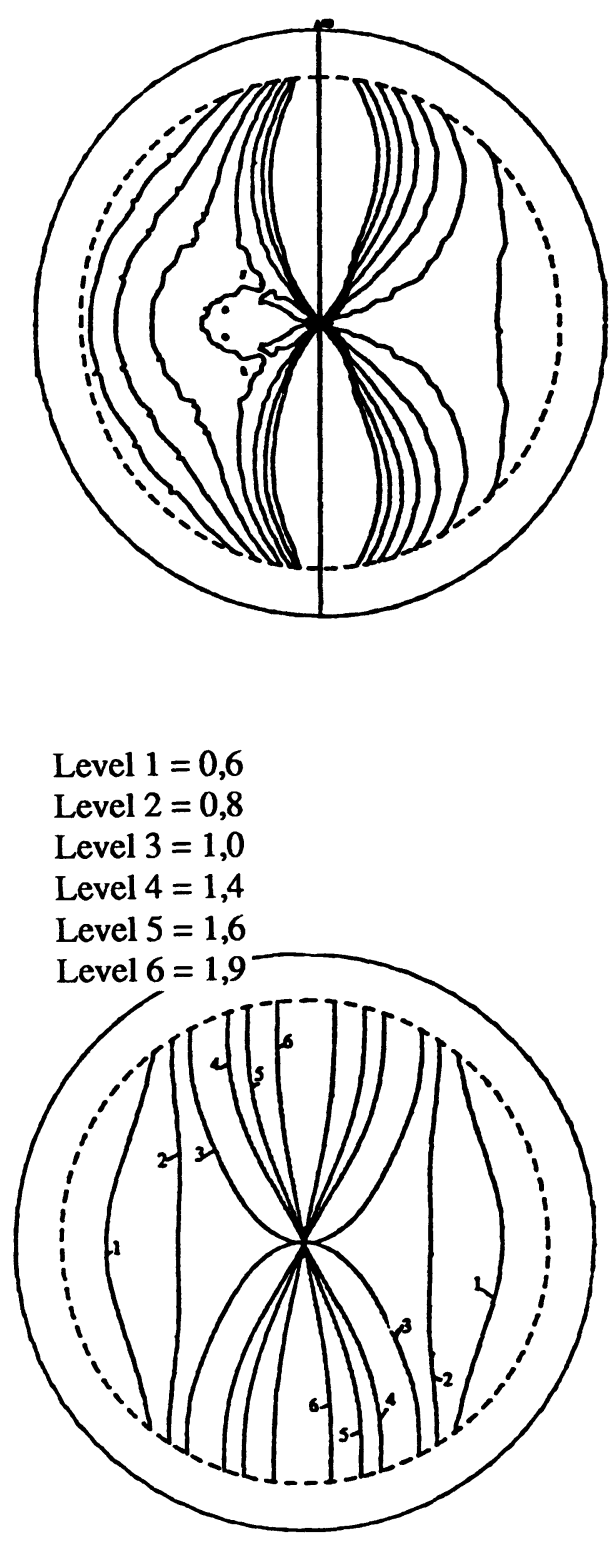$$
\text { . }
$$

Fig 6 a) (110) pole figure of a $0,3 \mathrm{~mm}$ wire coming from a $1,37 \mathrm{~mm}$ wire.

b) calculated pole figure of the corresponding correction factors.

c) (110) pole figure after correction. 